Jones, K. \& Edwards, J-A. (2017). Planning for mathematics learning. In S. Johnstone-Wilder, C. Lee, \& D. Pimm (Eds.) (2017), Learning to teach mathematics in the secondary school: A companion to school experience (chapter 5). Abingdon: Routledge. $4^{\text {th }}$ edition (pp. 70-91).

\title{
5 Planning for mathematics learning
}

\author{
Keith Jones and Julie-Ann Edwards
}

\section{Introduction}

Learning to plan effective mathematics lessons is one of the most important capabilities you can acquire in becoming a successful teacher of mathematics. Having a good lesson plan is significant for a whole host of reasons, not least in providing the structure which helps you to be confident that mathematics learning takes place during your lessons. Not only does good planning result in lessons that are interesting, challenging and motivating for your students, but also good planning is closely linked to the equally demanding (but often more overt) issue of effective classroom management. A good lesson plan, one that involves the class actively, helps to boost your classroom confidence and gives you a sound basis for managing the class successfully. Good planning goes a long way towards preventing classroom problems, by providing a structure that means that you can concentrate on managing learning effectively as your lesson unfolds.

Learning to plan good lessons takes both effort and time. This is because the success of your planning depends on your knowledge and understanding of a complex set of matters, including: how students learn mathematics; the structure of the mathematics curriculum; the specific content, techniques and concepts you are teaching; the prior knowledge of the students; ways of teaching mathematics; and how lessons can be planned for maximum effectiveness. Devoting sufficient time (and emotional energy) to planning is definitely worthwhile. It is a valuable investment for future years; it is an investment that, in the longer term, reduces the demands of paperwork and strain on your emotions, since your planning becomes quicker and easier as your experience grows.

This chapter addresses what it takes to plan mathematics lessons, covering the setting of learning intentions and how to structure individual lessons. This leads into how to plan sequences of lessons, including how to take account of students' prior knowledge and varying needs, how to select and prepare resources (including the use of digital technologies), and how to build in assessment opportunities. Following a look at the wider aspects of planning, including planning as part of a team and planning for out-of-school learning, the chapter concludes by looking at your developing expertise as you work towards becoming the creative and effective teacher that you wish to be (there is also appreciable discussion of these themes in general; see Capel, Leask and Turner, 2013, Unit 2.2). The chapter opens by looking at why planning is important, how it links with other aspects of teaching and how your lesson planning fits in with other levels of planning in the mathematics department in which you teach. 


\section{Objectives}

By the end of this chapter, you should be able to:

- understand the relationships among the mathematics curriculum, a scheme of work, the choice of teaching strategies and your individual lesson plans;

- $\quad$ select appropriate teaching strategies and mathematical tasks and resources (including the use of suitable digital technologies);

- $\quad$ plan mathematics lessons and units of work, identifying suitable learning intentions and content;

- $\quad$ set appropriate and demanding expectations for student learning;

- $\quad$ plan assessment opportunities;

- $\quad$ work as part of a team in your planning and plan for equity and out-of-school learning.

\section{Teachers planning lessons}

The demands of lesson planning can add to the burdens you feel as a beginning teacher if you are not clear about why lesson planning is necessary and exactly how it can help you in your role as a teacher.

\section{Task 5.1 Why is planning important?}

Write down five (or more) reasons for planning lessons. For each reason, explain why it is important.

In considering the reasons for planning lessons you may have written down some or all of the following:

\section{Lesson planning...}

- $\quad$ makes you articulate what you think will happen in a lesson;

- helps you to ensure that your lessons begin interestingly, maintain a good pace throughout and have a satisfying ending;

- enables you to rehearse various aspects;

- makes you more likely to be receptive to the ideas of others;

- $\quad$ provides a basis for negotiation, discussion and evaluation;

- creates a feeling of confidence for you, the teacher;

- provides a history of your thinking and development.

Or you might have written:

Lesson plans help to ...

- $\quad$ structure your lessons;

- build on previous lessons and learning; 
- $\quad$ share the learning intentions of the lesson with students;

- address the key questions you need to ask;

- $\quad$ highlight key vocabulary;

- make explicit the key teaching strategies you are using;

- focus on targets for raising the standard of achievement in the class;

- assess student achievement, so that you can take this into account in future lessons;

- develop effective 'assessment for learning' (see Chapter 7), so students receive feedback that helps them to improve;

- make lessons more inclusive and address a range of needs;

- make better use of classroom support and learning assistants;

- $\quad$ set homework.

You might like to compare your ideas about lesson planning with how Li, Chen and Kulm (2009) and Shen et al. (2007) describe lesson planning as integral to teacher professional development in China.

\section{What is a lesson?}

The conventional unit of teaching is the lesson - although in any given school a lesson might last anywhere from 30 to 70 minutes (or longer in a few cases). In contrast, there is no conventional unit of learning. Learning can take place at any time, day or night, and does not necessarily occur only in the presence of the teacher. Breaking down learning into lesson-size chunks is necessary for teaching, but this can result in a fragmentation of topics and ideas if teaching is solely thought of in terms of individual lessons. For example, without careful planning, students may not come to appreciate the connections between fractions, decimals and percentages, particularly if these are all treated separately. Another form of fragmentation can happen when investigative or problem-solving tasks are treated as comprised solely of distinct components such as 'generating results', 'drawing a table', 'finding a formula' or 'drawing a graph'. Effective planning has to be in terms of individual lessons that work well, but planning also needs to look to the longer term, so that individual lessons contribute to logical progression, fluency, reasoning and mastery in mathematical learning.

\section{Task 5.2 What are mathematics lessons like?}

The mathematics lessons that you observe in schools are likely to differ in form and approach. From your observations of different teachers and different mathematics lessons, record as many different formats as you can. How might the lesson plan (and the lesson planning) be different for different forms of lesson?

Devise a way of categorising the variety of mathematics lessons that you observe. In what ways do the teaching approaches and strategies vary? How does this variety fluctuate over time and with different classes?

Talk to teachers about how they have come to use particular strategies in the way that they do. Can any piece of mathematics be introduced to students in any way you choose or can you detect influences that guide teachers' choices of teaching strategy? 
There are a number of reasons why the mathematics lessons you observe may differ in character and approach. Partly, this may be due to individual teacher 'style', but there can be other underlying reasons. One thing to observe is that effective lessons have a structure. Typically, a mathematics lesson might consist of:

- a starter task (perhaps an oral and mental one) taking about 5 to 10 minutes;

- a major segment of whole-class and/or paired or group work (about 25 to 40 minutes) combining teaching input and student work on tasks;

- a final plenary (from 5 to 15 minutes) to round off the lesson (by summarising key facts and ideas, discussing the next steps, setting homework, etc.).

Of course, other lesson structures are possible. Below are examples of the structures of mathematics lessons from a study of mathematics teaching in the US and Japan on the topic of the area of triangles (Stigler, Fernandez and Yoshida, 1996):

\section{Typical US mathematics lesson}

teacher reviews concept of perimeter (1 minute);

teacher explains area of rectangle; students do practice examples (8 minutes);

teacher explains area of triangles; students do practice examples (25 minutes);

students work individually on an exercise (11 minutes).

Typical Japanese mathematics lesson

teacher presents a complex problem (4 minutes);

students attempt to solve the problem on their own or groups (15 minutes);

students' presentations and class discussion of student solutions to the problem, combined with teacher explanations, leading to general solution (21 minutes);

students work on practice problems (5 minutes).

In the example above from Japan, the 'complex problem' is sometimes called the 'problem of the day'; in other parts of the world it might be called the 'anchor task' (as it 'anchors' the lesson). While lesson structure is not the only influence on student achievement, it is worth noting that, in recent large-scale international surveys of mathematical achievement, Japanese students have scored amongst the best in the world in mathematics, whereas students in the US (and in the UK) generally score somewhat lower (see Jerrim and Choi, 2014; Jones, 1997). This suggests that what may influence how successful students are in mathematics is both how mathematics is taught (i.e., the teaching strategies that are used) and what forms of mathematical knowledge are taught (where the form of mathematical knowledge might be procedural or conceptual, or a combination).

\section{Task 5.3 How are mathematics lessons structured?}

From your observations of mathematics lessons, what different lesson structures are used? Are the lessons you see more typical of the US lesson or more typical of the Japanese lesson? 
In its reports Mathematics: Understanding the Score (Ofsted, 2008) and Mathematics: Made to Measure (Ofsted, 2012), Ofsted describes what mathematics lessons in England are like. You might like to consider how examples such as this, and examples from other countries such as those reported in Clarke, Keitel and Shimizu (2006) or Stigler and Hiebert (1999), might inform your ideas about how to structure your own lessons.

\section{How experienced teachers plan}

Whenever you observe effective teachers of mathematics at work, you observe the results of their planning. Research suggests that, in constructing lessons, experienced teachers draw on a range of experiences and knowledge in an attempt to match the anticipated and observed needs of their students to a particular lesson or set of lessons (John, 1993; Wragg, 1995). For experienced teachers, in many instances, lesson outlines consisting of phrases or illustrative diagrams are likely to be entirely appropriate. While these are fully meaningful to the teacher concerned, such lesson outlines may not conjure up a complete lesson to someone looking at such an outline (such as a novice or a substitute teacher). Alternatively, experienced teachers may appear to teach more or less directly from a textbook or from the departmental 'scheme of work' (see below), again making allowances for their particular classes. For a very experienced teacher, there are times when a fully detailed lesson plan may be appropriate (for instance, when a new or seldom taught topic is scheduled or as a useful basis for dialogue with a teacher colleague such as you), but such detailed plans are not always necessary for them.

This preference, by experienced teachers, for a more fluid mode of planning can appear to pose a difficulty for you. It can be that the more skilful the planning, or the more it happens at unscheduled times, the more difficult it is for you to understand how successful lesson planning is achieved or to 'see' the planning that underpins a lesson that you observe. What is more, the likely requirement on you that you produce detailed written plans as part of your training may seem oddly at variance with the practice of established teachers you encounter. Nothing could be further from the truth. All successful teachers carry out planning and it remains of critical importance. It is how you begin to plan, how you (and colleagues involved in your training) evaluate how these plans are enacted in the classroom and how your planning changes as you develop professionally that you should keep in mind.

Likewise, reflecting on your lesson plans as you use (and quite likely re-use) them also plays an important role in your development. Detailed written plans should give you the confidence to begin teaching well. As your confidence grows, and as you make progress with your classes, you might begin to reconsider the appropriate amount of detail to include in your plans.

\section{Task 5.4 How do experienced teachers plan?}

Ask a class teacher if they can take you through the process involved in planning a particular lesson. What are the important aspects of this process? What aspects of the plan are recorded? How are these aspects recorded?

See if you can observe, or take part in, the lesson and then discuss with the class teacher how the lesson went in practice. How and why might it have deviated from the lesson as planned? 
Pegg (1989) suggests a way of analysing a mathematics lesson. You could see how this might help you to better understand what is involved in planning mathematics lessons.

\section{How planning links to other areas of teacher knowledge and expertise}

By now, it should be clear that planning is a key professional responsibility and that your own planning is an important place in which you develop and show the high expectations you have of all the students you teach. Working with others, both in planning lessons and in learning from implementing lesson plans, is also central to developing and demonstrating professional values of the highest level.

In order to plan confidently and effectively, you need a high level of mathematical subject knowledge and understanding. This enables you to judge how mathematical ideas and concepts can be broken down and logically sequenced so they support students' mathematical learning, how you can make connections between mathematical ideas and how you might tackle likely student errors and misconceptions (for more on such issues, see Ryan and Williams, 2007; Watson, Jones and Pratt, 2013). What is more, lesson planning entails ensuring the purposeful use of a range of monitoring and assessment strategies, and using the information gathered to improve future planning and teaching.

\section{Levels of planning}

Planning can be thought of as operating at three levels of time-scale in relation both to the curriculum and to the individual or groups of students: long-term; medium-term; and shortterm. As a beginning teacher, you are understandably likely to be most focused on your short-term planning - what your classes are going to do tomorrow and the day after. This is tackled very soon in this chapter. At this point, it might help to recognise that the three levels of planning are intricately linked, as illustrated in Figure 5.1, and that what you do in your short-term planning accumulates to the medium term and then to the long term.

Keeping an eye on the medium term and, through that, on the long term is important if your students are going to progress in mathematics in the way you would like. Indeed,

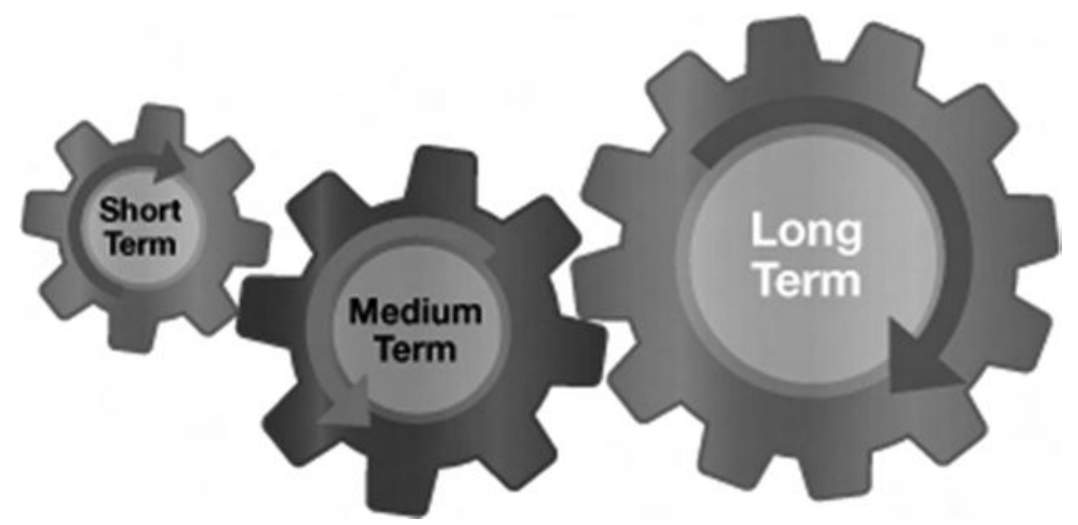

Figure 5.1 Links between the three levels of planning 
Jerome and Bhargava (2015) are of the view that being able to conceptualise your students' learning over a longer period of time (such as the medium term) is key to achieving outstanding learning as part of everyday teaching.

What follows is a short explanation of some inter-relationships among levels of planning. This leads into a consideration of sources such as the departmental 'scheme of work' (see below) and a national curriculum, and resources such as textbooks - and how these can help as you begin your own planning of individual lessons.

Long-term planning occurs at the departmental level and is informed by school-level policies and procedures. Such long-term planning addresses, among other things, how the statutory components of the curriculum are to be covered. In general, long-term planning at the departmental level is mainly coverage of the curriculum, with acknowledgement of students being referred to as year groups. As a beginning teacher, you normally work with the fruits of departmental long-term planning such as the 'scheme of work' (see Task 5.5).

Medium-term planning might be half-termly or termly or might possibly be by year. Such planning is often specified within a departmental 'scheme of work' (see Task 5.5). Here, a national curriculum document is likely to play a major role, as might a 'subject specification' for a national examination (such as the GCSE or A-level in England). Medium-term planning generally states the sections of the curriculum to be covered within the specific time-scale and addresses how this might be different for various sections of students within an age group (e.g., where there are students with special educational needs - see Chapter 11). Again, as a beginning teacher, you normally work with the fruits of departmental medium-term planning such as relevant sections of the 'scheme of work'.

Short-term planning is likely to encompass weekly, daily or individual lesson plans. Here, students become the primary focus, with small segments of the curriculum addressed for the various teaching groups. The planning of individual lessons, and how this leads to planning sequences of lessons, is covered in detail below. Before that is guidance on working with the departmental 'scheme of work' and the National Curriculum, as well as textbooks and existing resources in your planning.

\section{Working with the departmental 'scheme of work' and the National Curriculum}

\section{Task 5.5 What is a scheme of work?}

Examine a 'scheme of work' in a mathematics department and see to what extent it:

- $\quad$ provides a framework for classroom practice;

- details the knowledge, techniques and processes to be taught during a half-term or term;

- $\quad$ gives guidance about the range of teaching approaches to be adopted.

Now choose a topic or an area of work from the scheme of work in your school. Find out what the topic is designed to achieve, what the students were taught previously, what length of time is devoted to the topic, what resources are suggested, what use is made of digital technologies, the extent to which connections between areas of mathematics and beyond are addressed, how the work is assessed and what mathematics the students are to do next. 
Watson et al. (2013, p. 4) identify what they call seven 'key mathematical domains'. These are: relations between quantities and algebraic expressions; ratio and proportional reasoning; connecting measurement and decimals; spatial and geometrical reasoning; reasoning about data; reasoning about uncertainty; functional relations between variables. You might find this helpful in identifying how the departmental 'scheme of work' in the school in which you are working relates to the National Curriculum and how this relationship can aid your lesson planning.

As noted above in the sub-section on levels of planning, the structure of national (or local) curriculum documents needs to be taken into account when learning to plan. For example, for England (as currently specified) there are two aspects that are of central importance to your planning; namely, the over-arching aim of 'working mathematically' (with the themes of fluency, mathematical reasoning and problem solving) and the subject content (which identifies the mathematics to be taught). One way to think about the relationship between the two is to consider the subject content as indicating what you should plan to teach, while the themes within 'working mathematically' indicate how you might plan for developing interconnections between areas of mathematics and beyond for your students. It is important that what happens during your lessons provides the link between the two.

\section{Task 5.6 How can the mathematics curriculum aid lesson planning?}

Choose two statements from different content areas of the National (or local) Curriculum document for mathematics (such as the Programme of Study for Key Stage 3 Mathematics for England) and examine the themes of fluency, reasoning mathematically and solving problems as these appear in the chosen content areas.

For each of the chosen curriculum statements, identify rich connections with other statements in the subject content that you think would allow students to experience different representations of mathematical ideas. How do these connections appear in the scheme of work in the mathematics department in which you are teaching?

Watson et al. (2013, p. 5) claim that, 'some key ideas in mathematics pervade the whole of the subject and are integrated into many chapters [of the book]', adding that, 'this is true of proof, ICT use, and representation [of mathematical ideas]'. You might like to consider how mathematical representations influence what you consider when you plan lessons.

It is worth noting at this point that the current version of a national (or local) curriculum for mathematics is only one vision of how the mathematics curriculum can be specified. For example, while it is common for the mathematics curriculum for schools to be itemised in terms of content such as 'number', 'algebra' or 'probability', other ways might include arranging the curriculum around what are sometimes referred to as the 'big ideas' in mathematics such as place value, variable, function, invariance, symmetry, proof and so on (see Watson et al., 2013, pp.4-8). The resulting curriculum might well appear very different and, as a consequence, perhaps be taught in a different way. This illustrates the idea of the curricular shaping of teaching: that is, how the specification of the curriculum directly influences the teaching strategies used.

\section{Using textbooks and existing resources in planning}

Textbooks can offer a structure to guide you when planning a lesson or series of lessons. Textbooks may also reveal aspects of a topic and suggest contexts for teaching that you may 
not have considered. They may save you time by providing a suitable task that you could use 'as is' or adapt for your classes. In some countries, Japan being an example, textbooks are the result of continual improvement over many years and can be considered to capture 'best practice' in mathematics teaching. In other countries, textbooks can vary considerably in format and quality; some, for example, may consist primarily of sets of exercises, while in others each section focuses on explaining and practising a specific mathematical procedure. Given this variety, judicious use of a suitably chosen textbook can provide helpful introductory scenarios for lessons, can give ideas for ways to structure a logical progression of mathematical ideas and can provide tasks and exercises that can help to develop and consolidate student learning towards mathematical reasoning, fluency and mastery.

Takahashi (2011, p. 216) explains how, in Japan, the view is that good teachers know how to 'read between the lines of the textbook'. You might like to consider how you might initially 'teach the textbook', to use Takahashi's term, and how you might develop so that you are 'using the textbook to teach mathematics' (p. 201).

Increasingly, there are online repositories that provide access to a wealth of resources for teaching. As with some textbooks, the 'quality assurance' mechanisms for such repositories can be unclear; hence the quality of the resources can be somewhat variable. While online repositories are readily accessible, it can be all too easy to spend excessive time searching for a 'perfect' resource for the learning intention(s) you want to reach or a resource that is 'just right' for your class. Developing a sense of what is likely to 'work' with your classes can entail trying things out (and taking a few risks) with the guidance of an experienced teacher. Further below is a sub-section devoted to working with existing lesson plans (and resources) and before that is the section that begins with the first steps in lesson planning.

\section{Planning a lesson}

A good place to begin your first steps in lesson planning is with a reasonably self-contained part of a lesson with which you are likely to feel comfortable. This could be one or more of the following:

- $\quad$ taking a lesson 'starter';

- $\quad$ presenting an agreed segment of the main part of a lesson;

- concluding a particular lesson.

Each of these ways of beginning planning and teaching needs to be negotiated and agreed with the class teacher. All are opportunities to boost your confidence with speaking to a whole class and may help you to get to know the names of individual students. Planning for these first steps might well involve specifying precisely what you say, based on the class teacher's lesson outline (or the departmental scheme of work). Here you might prepare a 'script' of what you plan to say.

\section{Task 5.7 Planning and presenting parts of lessons}

Negotiate to take a reasonably self-contained part of a lesson. Discuss your plan for this lesson segment with the class teacher. What aspects of the lesson segment would you like feedback on? Afterwards, discuss how the segment went in practice. Which aspects of your planning and presentation do you need to work on? 
Zazkis, Sinclair and Liljedahl (2013) use the term 'lesson play' to refer to a lesson (or part of a lesson) prepared in the form of a script that features the imagined interactions between a teacher and the students. You might like to try out the idea of a 'lesson play' when you are planning part of a lesson and see how this might help you to anticipate what might happen when you actually teach the lesson segment.

\section{Planning whole lessons}

Planning whole lessons entails specifying some or all of the following:

- $\quad$ the learning intentions and resources for a 'starter' task (perhaps some oral and mental mathematics);

- the learning intentions to be addressed in the main teaching tasks and related student activity;

- the key teaching points and tasks for the lesson, matched to the learning intentions and with suggestions for how the main idea could be developed;

- the rough timing of each element of the lesson;

- $\quad$ key mathematical terms and notation to be introduced/used;

- the resources needed, including ICT, departmental resources and sections of textbooks;

- $\quad$ ideas to be drawn out in the plenary (or mini-plenary) sessions, including some key questions and homework tasks;

- opportunities for assessing (usually formatively) how successful the students are with learning the key ideas in the lesson.

\section{Task 5.8 Beginning planning whole lessons}

Negotiate with the class teacher that you work together to plan a complete lesson that the teacher takes. While the class teacher is teaching the lesson, make notes on the opening, middle and closing segments of the lesson, the timing of each segment and any deviations from the lesson plan. Discuss with the teacher how each segment went and the reasons why the teacher decided to make any changes or adjustments.

Negotiate to plan further lessons with the class teacher for which you will undertake agreed elements. As noted above, you could, for example, start the lesson or conclude it. Discuss with the class teacher the extent to which the learning outcomes for the lesson were achieved. How can you build assessment opportunities into your plans and use the outcomes to inform your future planning?

One way to approach planning whole lessons is to use a template (or pro forma). An example is shown (complete with lesson plan) in Figure 5.2. These usually contain many of the elements explored earlier in this chapter, such as:

- $\quad$ practical details such as date, class, time, room;

- reference to topic, module or scheme of work;

- aims, learning intentions or learning outcomes; 
- teacher tasks and tasks for the students;

- timings for elements of the lesson;

- homework.

More advice is provided by Jerome and Bhargava (2015), John (1993) and Savage (2015).

\section{Task 5.9 Using lesson planning templates (also called pro forma)}

Review some of the lesson templates (or pro forma) that you have seen or have been given (see the example in Figure 5.2; there are more examples in John, 1993). How suitable are they for the lessons you teach? How might using different templates aid the planning of your lessons? In what ways might particular templates restrict or limit how you work with your classes?

An alternative to using pre-designed templates (or pro forma) is to design your own. Try designing one or more templates for your own use. How do the templates you design vary according to the format or learning intentions of a particular lesson? What essential elements should a lesson-planning template contain?

Theoharis and Causton-Theoharis (2011, p. 747) found that a pre-designed template needed to be carefully organised if its completion was to avoid being 'lengthy, overwhelming, extremely detailed [such that] it seemed formidable to plan in this manner'. You could consider how you decide the appropriate balance in your lesson planning between essential detail and overwhelming detail, and whether the amount of detail varies with the class and the lesson topic.

An important aspect of successful lesson plans (and hence successful lessons) is careful consideration of the various stages in a lesson. It is certainly likely that some lesson segments will take longer than you expect, while others may take considerably less. Clearly, you will need to adjust your plans to account for both eventualities. This involves working flexibly with your lesson plan and having more ideas at hand in case you need them. Always ensure you finish on time and avoid rushing things if they are taking longer than you expect. If the main task needs more time than you allocated in your plan, it can be better to cut out part of it and keep it for next time and have a proper finish - rather than have a lesson end abruptly and unsatisfactorily.

\section{Selecting learning intentions}

As John (1993, p. 30) claims, 'virtually all major guide books on curriculum and lesson planning begin with the importance of laying down, at an early stage, the educational and learning goals that will guide the lesson'. This reflects the view that the way to introduce beginning teachers to the complexities of lesson planning is to use a framework based around the 'rational planning model' first outlined by Tyler (1949). This model asserts that planning a lesson or a sequence of lessons involves:

- $\quad$ specifying objectives;

- $\quad$ selecting and sequencing learning tasks;

- $\quad$ evaluating the outcomes. 
The advantages of clearly specifying objectives include that they:

- $\quad$ are easily communicated;

- help to clarify thinking and planning;

- are measurable;

- $\quad$ are linked to observable classroom outcomes;

- make assessment and evaluation clearer.

While it is undoubtedly important to be clear about what students are to learn during a lesson, there are disadvantages to starting the process of lesson planning with a set of lesson objectives. Amongst the disadvantages are:

- $\quad$ planning can become more rigid;

- opportunist learning might be inhibited;

- learning outcomes might be trivialised;

- $\quad$ alternative or additional learning occurring during the lesson can be overlooked;

- a technicist rather than a more creative view of teaching is likely to be encouraged.

When thinking about lessons, and especially when using lesson templates or pro forma (whether pre-designed or designed by you), it helps to learn how to work creatively with learning intentions (the latter term is used by Wiliam, 2011, in preference to 'objectives'). Unless you work creatively with learning intentions, research evidence suggests that there is a real risk that planning will become overly rigid and thereby inhibit learning opportunities that can arise (John, 2006; Wiliam, 2011). Working creatively with learning intentions means, among other things, looking for interesting tasks and deciding how these match with what you are teaching (rather than always starting with the learning intention and trying to find tasks that match it).

While specifying learning intentions concentrates attention on what is to be taught, and how students' learning will be judged, what may not be addressed is the important issue of how students will be provided with learning opportunities. What matters is that there should be some criteria for how success - or degrees of success - might be evaluated, and this is where learning intentions are important.

Where possible, learning intentions should be precise, assessable and achievable and, as such, be a statement of learning outcomes in terms of students' learning, not what the students will be doing as they complete a task. In this way, learning intentions should help to frame the lesson (or sequence of lessons) and aid the articulation of 'key' questions to inform the structure of an introduction or conclusion. Learning outcomes can also be used as assessment criteria, with useful 'stems' for learning intentions, beginning 'By the end of the lesson students will ...' (or equivalent phrases).

\section{Task 5.10 What is involved in specifying learning intentions?}

Review some of the successful lessons you have seen. How easy is it to specify the learning intentions for each one? Are some sorts of learning intentions easier to specify than others? Is it always appropriate to share learning intentions with students? And always before the lesson starts or only at the end? How can you tell to what extent your learning intentions for your students have been met? 
John (2006, p. 483) argues that the 'rational planning model' leads to 'a limited view of teaching and learning as well as a restricted approach to learning to teach'. You might like to take a look at his suggestion for 'an alternative, dialogical model of lesson planning' that 'not only emphasizes context-dependency but also sees planning itself as a practice' and see how this might inform your approach to lesson planning. For further research-based advice on working with learning intentions, see Chapter 3 of Wiliam (2011).

\section{Working with existing lesson plans}

Working with existing lesson plans, such as the one in Figure 5.2, and modifying them to the particular circumstances of the class you are teaching, provides another way to begin learning how to plan lessons. Increasingly, there are online sources of lesson plans, although, as noted above, the quality can vary and you can be drawn inadvertently into spending a

\begin{tabular}{|c|c|c|}
\hline Year 7 & & $\begin{array}{l}\text { Topic: Fractions } \\
\text { Curriculum reference: Number }\end{array}$ \\
\hline \multicolumn{3}{|c|}{ Key vocabulary: numerator denominator proper fraction equivalent fraction } \\
\hline \multicolumn{3}{|c|}{ Starter learning intention: ways to express one quantity as a fraction of another } \\
\hline $10 \mathrm{~min}$ & Starter Task & $\begin{array}{l}\text { Use whiteboards or digit cards } \\
\text { Ask questions such as: } 18 \div 3,20 \div 5.1 / 4 \text { of } 80,1 / 10 \text { of } 70,2 / 3 \text { of } 24,4 / 5 \\
\text { of } 40 \\
\text { Discuss methods }\end{array}$ \\
\hline \multicolumn{3}{|c|}{$\begin{array}{l}\text { Main lesson learning intentions: represent fractions on the number line; find differences between } \\
\text { pairs of fractions; recognise equivalent fractions }\end{array}$} \\
\hline $15 \mathrm{~min}$ & $\begin{array}{l}\text { Introduction } \\
\text { and Task }\end{array}$ & $\begin{array}{l}\text { - With a } 60 \mathrm{~cm} \text { line on the board, model the task by throwing two dice } \\
\text { to get the numbers to make a proper fraction. Plot the fraction on the } \\
\text { line. Repeat several times. } \\
\text { - With students in pairs or small groups, on large paper, each draws } \\
\text { a line } 60 \mathrm{~cm} \text { long. Using two dice, students create random proper } \\
\text { fractions and mark them underneath their number line. } \\
\text { - Ask pairs to put in any other fractions with denominators 2, 3, 4, 5, } 6 \\
\text { (without rolling the dice) (Key Question: How many will there be } \\
\text { altogether?), then ask pairs to plot tenths and eighths. }\end{array}$ \\
\hline \multirow{2}{*}{$15 \mathrm{~min}$} & Extension & Plot sevenths and ninths as well; further extension to improper fractions \\
\hline & Equipment & $\begin{array}{l}\text { Large sheets of paper with } 60 \mathrm{~cm} \text { line drawn } \\
\text { Dice (6-sided or } 12 \text {-sided) } \\
\text { Whiteboards or digit cards }\end{array}$ \\
\hline Plenary & & $\begin{array}{l}\text { Look at results and ask did you find any equivalent fractions? } \\
\text { Key Questions: } \\
\text { Which is larger: } 2 / 5 \text { or } 1 / 3 ? 3 / 4 \text { or } 4 / 5 ? 2 / 3 \text { or } 7 / 10 ? \\
\text { Which pair of fractions has the bigger difference: } 3 / 5 \text { and } 4 / 6 \text {, or } 4 / 5 \text { and } \\
5 / 6 \text { ? } \\
\text { How could such results be explained using equivalent fractions? }\end{array}$ \\
\hline
\end{tabular}

Figure 5.2 An example lesson plan (that uses a basic pro forma) 
disproportionate amount of time searching for the 'perfect' lesson plan that may not yet exist - indeed, the plan in Figure 5.2 is not meant to be 'perfect'.

With an existing lesson plan, a first step is to work through the plan and consider what the lesson is aiming to achieve and why it is structured in the way it is. You could try this with the lesson plan in Figure 5.2. For instance, the main part of the lesson (featuring what is sometimes called the 'anchor task') is predominantly group work; you could see how the plan could be adjusted to include students presenting their work to the class. If an extension or plenary question was 'Which is bigger 201/301 or 200/300?', you might consider how that could influence the thrust of the lesson. You could also consider how the lesson might be reinforced by homework and what might be a suitable subsequent lesson.

Modifying existing lesson plans is an important skill. It entails matching the lesson to what you know of the students you are teaching. Will they find the lesson engaging and motivating? Challenging? What will they learn? Does the context need adjusting? Is the timing of the various segments suitable? These are the sorts of questions to ask yourself when you are modifying existing lesson plans.

\section{Task 5.11 Modifying existing lesson plans}

Find some existing lesson plans that are suitable for a topic you are planning to teach and try modifying one or more of these for the students you are teaching. Which elements of the existing plans can you keep? What might need adjusting to match the needs of your students? How will you decide?

Li et al. (2009) show how six different teachers modified a lesson on fraction division to suit their classes. You could see how the various lesson plans from the six teachers were similar in terms of some broad features, but differed in detail and specific approaches used.

\section{Choosing, modifying and designing tasks}

Choosing tasks, modifying them and perhaps designing them all constitute part of effective lesson planning. Particularly important are the choice of starter task and, especially, the choice of 'anchor task' - the key task featured in the lesson. While, as noted above, increasingly there are online repositories that provide access to a wide range of examples of classroom tasks, often provided by practising teachers, even then the quality can vary. A well-designed textbook can be a good source.

Mason and Johnston-Wilder (2006) provide a way of approaching the design of mathematical tasks. They include several 'frameworks' for thinking about tasks, as well as ways to consider what form of task might start a new topic or what task might conclude a topic. Look out for mathematical tasks that use prompts such as the following: 'Always sometimes never', 'What's the same, what's different?' or 'Odd one out' to focus on mathematical reasoning, fluency and mastery. Work on ways of including these forms of task in your lesson planning and how the idea of 'Teaching with variation' helps with 'intelligent practice' (see NCETM, 2015). Find ways of sharing and discussing ideas for starter and anchor tasks with other mathematics teachers. 


\section{Reflecting on your lesson plans and developing better ones}

Reflection on all the points raised in this section will provide you with the basis for sound evaluation of your work and of your progress as a developing teacher. When reflecting on your planning of individual lessons, you will probably concentrate on:

- What was the best thing about the lesson?

- What did I enjoy most? What did the students enjoy most?

- How did the students react to the lesson? Why?

- What would I change about the lesson if I did it again?

- At what points in the lesson could I have engaged the students more? How?

- Were the students able to do what I wanted them to do? Why/why not?

- What did the students learn? In planning for what would likely be the 'difficult point' during the lesson, was I right?

- Did the students reach the learning intentions for the lesson? Why or why not?

As you become more experienced, your lesson plans can become more developed and might also include:

- potential difficulties or misconceptions that students may have, and possibilities to preempt or rectify them;

- $\quad$ how best to deploy any available support staff;

- connections with other mathematical topics and other subjects;

- additional assessment strategies.

Yang and Ricks (2012) explain that teachers in China have developed a framework called 'Three points' to help them reflect on how their lesson plans have gone in practice in the classroom. These three points are: the lesson's key point (the central learning intention of the lesson that the students must grasp); the lesson's difficult point (the cognitive difficulty that students might encounter as they try to learn the mathematical key point); and the lesson's critical point (how to help students eventually reach the key point while avoiding or overcoming the pitfalls that might arise because of the difficult point).

While not necessarily being explicitly shown in lesson plans, the three points are important to teachers in China. This is exemplified by Chen and Leung (2015, p. 543) who quote a mathematics teacher in China explaining that "the most important thing in a lesson is to enable the students clearly to know what are the "essential [key] points" and "difficult points" of this lesson, and to understand these points through [my] explanation'. As you reflect on your lesson plans, and begin developing your expertise, you might find it useful to consider each of your lesson's key point, difficult point and critical point. Working collaboratively with other teachers and educational professionals on ideas such as this is the theme of the next section.

\section{Planning collaboratively}

As well as working collaboratively with an experienced teacher, as a beginning teacher you may have the benefit of being paired with another beginning teacher. Such arrangements provide many opportunities for collaborative planning, teaching and reflecting. For more 


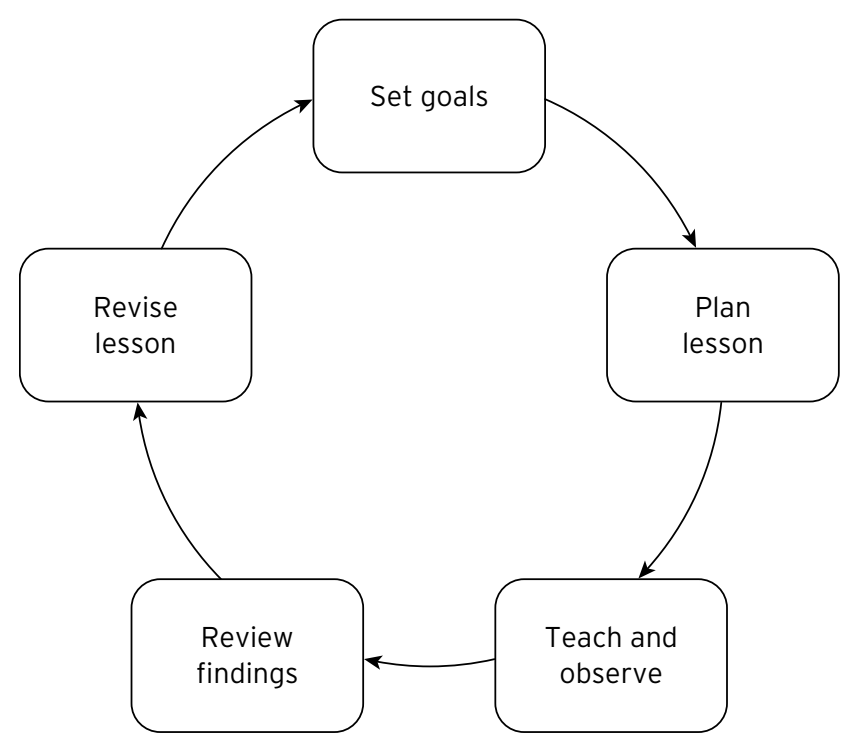

Figure 5.3 The lesson study 'cycle'

information on the 'paired placements' of beginning teachers, see Smith, J., 2004, and for more on the idea of 'co-teaching', see Roth and Tobin, 2005.

Other examples of ways of collaborative planning take the form of 'lesson study', an approach developed in China and Japan over many years and now being used far more widely (see the range of examples in Hart, Alston and Murata, 2011). As Murata (2011, p. 2) explains, the idea of 'lesson study' is relatively straightforward and generally takes the following form: 'teachers come together with a shared question regarding their students' learning, plan a lesson to make student learning "visible", and examine and discuss what they observe'. As such, lesson study typically follows the steps shown in Figure 5.3, with what is usually called the 'research lesson' (the lesson observation) as the centrepiece of the study process.

While the idea of lesson study is relatively straightforward, in practice there is more to it than that. The evidence provided by books such as ones by Hart et al. (2011) or by Dudley (2014) shows not only the recognised benefits of lesson study, but also some of the obstacles that may need to be overcome. If you can gain positive experiences of such collaborative planning, teaching and reflecting, there is likely to be much that you will learn.

\section{Planning sequences of lessons}

Being confident about planning and leading individual lessons is a good start. Building on this so that you can plan and teach a coherent series of lessons allows you to tackle some of the problems associated with having to divide learning into lesson-sized chunks (where the danger is that mathematics can seem fragmented and incoherent for your students). This section looks at how you can ensure the continuity and logical progression of student learning in your classes, so that learning does indeed happen over time.

In this context, a topic of work is a coherent series of lessons on a mathematical topic (such as fractions or solving equations), on a 'big idea' in mathematics (such as invariance 
or symmetry) or a piece of project work (such as an investigation or a sizable open problem). The scheme of work in your department may specify quite precisely each lesson within a topic of work. Alternatively, it may provide no more than a title (or list of learning intentions) and a list of suggested resources. No matter what you have in your current circumstances, there are inevitably going to be times when you want to plan or review a topic of work.

Given that mathematics does not solely comprise a list of isolated learning objectives, but is more an interconnected web of ideas, it is the connections between these ideas that may not be obvious to your students. Good topic planning means trying to ensure that mathematical ideas are presented in an interrelated way. This means, as far as possible, planning to:

- $\quad$ present each topic as a whole - for example, showing students that decimals and percentages are particular forms of fractions or by ensuring algebraic links are made among tabulation, graphical and symbolic approaches;

- bring together related ideas across strands - for example, linking ratio and proportion in number to rates of change in algebra, to enlargement and similarity or to trigonometry in geometry and to proportional thinking in statistics and probability;

- help students appreciate that important mathematical ideas permeate different aspects of the subject - for example, linking the concepts of inverse and order in the four number operations to the transformation of algebraic expressions and the geometrical transformations of reflection, rotation and translation;

- use opportunities for generalisation, proof and problem solving to help students to appreciate mathematics as a unified subject.

Your plan for a topic should include:

- $\quad$ at least one key aim for the topic as a whole;

- an indication of progression; that is, what topics should come before and what might come after the topic being considered, as well as interconnections with other topics and other subjects (often detailed in the scheme of work);

- lesson plans for each lesson, detailing learning intentions for each individual lesson, outline starter tasks, how the work is to be developed in the main part of the lessons through teaching input and student activity, how each lesson is to be concluded, suggestions of what homework should be set and so on;

- details of relevant resources, such as textbooks, practical equipment, worksheets and digital technologies.

When selecting learning tasks it is a good idea to reflect on what makes an interesting, motivating and challenging one for your students. Consideration here includes deciding about:

- $\quad$ context ('real life', 'pure', 'imaginary', etc.);

- $\quad$ single outcome or many possible outcomes;

- ways of working:

- exposition by the teacher;

- discussion (small groups, whole class); 
- solving problems;

$\circ \quad$ investigating.

In terms of aims and learning intentions, it is probably wise to determine a general aim for the topic and, subsequently, determine more specific learning intentions for each lesson. Selecting these learning intentions involves separating a topic into distinct elements (or aspects) and designing a sequence through these elements. Research suggests that this is the most demanding aspect for a beginning teacher (see John, 1993, 2006). Deciding how to select learning intentions demands good subject knowledge, skill at separating and sequencing the elements of a topic, and awareness of student needs (for more advice, see Wiliam, 2011).

One way to begin planning topics is to rely on established practice (e.g., the scheme of work, a good textbook or equivalent, or an existing sequence of lesson plans). However you begin, remember that a learning intention is not about which exercises the class is to do, or what the students are going to write down, or even that they are going to have a class discussion. These are the actions and resources that you will be using to promote learning. Learning intentions specify what is to be learnt. A major pitfall in planning a topic is to neglect learning intentions and to see planning as simply organising tasks or actions; there is much more to it than that.

Curriculum documents can provide guidance on meeting curriculum requirements for mathematics. They may set out subject content to be taught, which can be used to help specify learning intentions in planning for teaching mathematics. In addition, they may specify aspects of working mathematically, which can guide you on how you might approach achieving interconnectedness in your students' learning. When planning for younger secondary school students, it is worthwhile looking at the curriculum specification for the upper primary years as well, so that you are guided by the progression that is identified there.

In terms of monitoring and assessing student progress, it is worth asking yourself if this is to be formal (say, through a test), informal (say, through your observations) or a mixture? It certainly needs you to be active and purposeful in the classroom (not just waiting for hands to go up, for example) and involves you observing, probing, questioning, checking, evaluating and so on. Planning for assessment means using assessment for learning to help students to reflect on what they already know, reinforce the learning being developed and set targets for the future. Planning for summative assessments (from end of topic tests through to yearly school tests and then relevant national tests) also needs to be part of planning. Assessment issues, including a discussion of assessment for learning and summative assessment, comprise Chapter 7.

\section{Task 5.12 Planning a topic}

For a topic you are beginning to plan, look at how the topic links to other topics and other subject areas, and how students' learning progresses in this area. What prior processes, techniques and understanding do they need to have to do the work on this topic? What similar topics will they have studied in previous years? What do they need to learn now in order to prepare them for later topics?

(continued) 
Task 5.12 (continued)

Look for suitable resources. How can you plan to include a range of tasks (including open-ended work, group work and individual work)? What tasks do you think your students will find interesting (and why)? How can you use a range of teaching and learning approaches? Will you need to create some of your own resources? What misconceptions may students have about this topic and how will you overcome them? What is each lesson's key point, difficult point and critical point? What assessment opportunities can you build in to your lesson plans and how will you record students' achievements and progress during this topic?

Jerome and Bhargava (2015) provide further advice on medium-term planning. You might see what you can learn from their advice.

\section{Planning for inclusion and equity}

The promotion and realisation of equity of opportunity for your students to learn mathematics needs to be integral to your planning. This entails taking care to promote student experiences, resources and content that do not reinforce - and wherever possible positively counteract forms of stereotypical thinking. It means ensuring that you provide the best for every student, irrespective of gender, social class or ethnicity. Each school is likely to have policy on how funding such as the 'Student premium' (or equivalent), or funding for those with 'special educational needs' (SEN), is used to support specific students (for more on this, see Chapter 11). It is important that you are aware of the school policy in planning for these students' learning.

While you, as the teacher of the class, have overall responsibility for student learning, the role of another adult in the classroom, such as a teaching assistant, can be open to some negotiation or might be quite clearly prescribed within the school - the latter is often true for teaching assistants who work with students with identified 'special educational needs'. It is good practice to liaise with the other adults in advance to ensure lesson planning is consistent, and shared with them.

\section{Task 5.13 Planning for inclusion and equity}

Review some of the successful lessons you have seen. How has the variety of students in the classes been included in the class activity? How does the teacher ensure that their teaching means that all students are achieving their best?

Talk to some experienced teachers about the best ways of planning when you have other adults in a class you are teaching. How might you involve the other adults, or brief them about your planning? How can your planning make sure that they are clear about roles in the lesson? How might you learn from their expertise?

Sharples, Webster and Blatchford (2015) make some suggestions about the ways of working with teaching assistants. You could take a look at their advice and see how this might influence how you plan your lessons. 


\section{Planning for out-of-school learning}

When planned well, homework experiences can provide a valuable supplement to classroom activity. For example, you can use homework to reinforce and consolidate classroom learning or to gather information that you will then use in the classroom. Homework is one way in which parents come to know about what their child does in your lessons. One particular issue to consider in your planning is whether you can use homework as an opportunity to practise techniques or for widening the perceptions of mathematics. Or perhaps, over time, you can aim to do both.

Valuable student learning can take place in a wide range of out-of-school contexts. Such opportunities are known to have positive effects on the achievement of students in mathematics (see Barbeau and Taylor, 2009). Examples of out-of-school learning can involve various partners such as museums, galleries, libraries, sports clubs, theatres and so on, plus opportunities for students to take part in mathematical competitions of various types. It can equally involve planning mathematics learning for a trip to a local park, a playground or even a cemetery to collect data.

Maloney et al. (2015) report that when parents who are mathematics-anxious help their children with their mathematics homework, their children can also become anxious about mathematics. Wager (2012) identified several ways in which primary teachers incorporated their student's cultural and out-of-school mathematics into their teaching. You might see how such research informs how you might inform your planning.

\section{Task 5.14 Planning for homework and other out-of-school learning}

What are some good ways of using homework, either to consolidate classroom learning or to gather information that you will then use in the classroom? What are the benefits of home-school contracts and how might you involve parents in supporting student learning? Find out about how to plan for out-of-school learning, including school policy on this, at the school where you are teaching. What mathematical competitions are available? What are some other ways in which you can plan for students to learn in out-of-school contexts?

Find out about the use of 'home-school contracts' (or equivalent) and how mathematics departments in particular, but schools more generally, involve parents in supporting student learning and helping towards addressing potential mathematics anxiety at home.

\section{Developing expertise for creative teaching}

As noted at the beginning of this chapter, experienced teachers may display a more fluid mode of planning. What is more, they may freely deviate from their lesson outlines during particular lessons as they seek to maximise the learning of their students.

One reason they can do this is that, through their expertise and through devoting considerable time to lesson planning during the early stages of their career, they already have lesson 'segments' in mind that they can creatively and quickly assemble into coherent and effective lessons. As Sawyer (2011, p. 3) explains, teacher expertise entails weaving together 
'a large knowledge base of plans, routines, and structures, within improvised practice'. In teaching mathematics, as Martin and Towers (2011, p. 275) go on to describe, the 'teachable moment' - when students can learn such a lot - is 'always unpredictably emergent'; indeed, successfully taking advantage of teachable moments requires 'a teacher who is adept at enacting improvisational interventions' (for more ideas about developing your planning, see Aberson and Light, 2015, and McCrea, 2015). As your expertise develops, and your planning becomes more skilful, you are honing your technique towards that artful balance of structure and improvisation that is the hallmark of creative effective teaching.

\section{Summary}

Your success in teaching depends crucially on the effectiveness of your planning and how well you put your plans into action. The lesson planning that you do needs to be explicit and detailed, particularly in the early stages of taking over classes. This takes good organisation and it takes time. Developing a range of lesson structures and matching these to what you want to achieve in your lessons is vital.

Spend time getting to know the structure of the curriculum, the departmental scheme of work and the resources already available to you, so that you can begin to work creatively within the statutory framework. Develop skill at making presentations, both verbal and non-verbal. Get to know what your classes can do and what motivates them. Build up a collection of classroom tasks that you are confident will engage the attention of students. Review and evaluate your work and both seek and act on advice. Find ways of collaborating with other teachers and other education professionals. Engage with research. Always expect a high standard of work.

Successful planning entails preparing a rich mathematical diet for your students. Your efforts will be rewarded with the quality of student learning you engender, the motivation of your students to learn mathematics and your own sense of achievement.

\section{Further reading}

Atkin, K. (2015) Presentation Workout: The 10 Tried-and-Tested Steps That Will Build Your Presenting Skills, Harlow: Pearson.

This guide covers many of the techniques involved in developing presentation skills that help to put a good lesson plan into action.

Jerome, L. and Bhargava, M. (2015) Effective Medium-Term Planning for Teachers, London: Sage.

This book shows how to conceptualise learning over a longer period of time and argues that this helps to support you to achieve outstanding learning as part of your everyday teaching.

Mason, J. and Johnston-Wilder, S. (2006) Designing and Using Mathematical Tasks, St Albans: Tarquin.

As well as suggesting ideas for mathematical tasks, this book provides a way of thinking about the design of tasks for students to help develop their mathematical thinking.

Neill, S. and Caswell, C. (1993) Body Language for Competent Teachers, London: Routledge.

Despite having a rather odd-sounding title, this book shows how to 'read' classroom situations and how to establish good relationships with classes. It contains many illustrations of classroom situations. 
Oldknow, A., Taylor, R. and Tetlow, L. (2010) Teaching Mathematics Using ICT, 3rd edn, London: Continuum.

This is a compendium of ideas and advice, including a chapter on how to plan effective use of digital technologies.

Savage. J. (2015) Lesson Planning: Key Concepts and Skills for Teachers, London: Routledge.

This book provides a general guide to lesson planning.

Watson, A., Jones, K. and Pratt, D. (2013) Key Ideas in Teaching Mathematics: Research-Based Guidance for Ages 9-19, Oxford: Oxford University Press.

This is a research-informed guide to teaching the main ideas in school mathematics.

\section{Acknowledgement}

We would like to record our thanks and appreciation to the many teachers, both beginning and experienced, with whom we have worked over the years and from whom we have learnt so much. 